\title{
Galactic and Magellanic Evolution with the SKA
}

\author{
Naomi M. McClure-Griffiths*1, Snežana Stanimirović ${ }^{2}$, Claire E. Murray ${ }^{2}$, Di Li $^{3}$, \\ John M. Dickey ${ }^{4}$, Enrique Vázquez-Semadeni ${ }^{5}$, Josh E. G. Peek ${ }^{6}$, Mary Putman ${ }^{6}$, \\ Susan E. Clark ${ }^{6}$, Marc-Antoine Miville-Deschênes ${ }^{7}$, Joss Bland-Hawthorn ${ }^{8}$, Lister \\ Staveley-Smith ${ }^{9}$, on behalf of the $\mathrm{H}_{\text {I }}$ Science Working Group \\ ${ }^{1}$ CSIRO Astronomy \& Space Science, Australia; ${ }^{2}$ University of Wisconsin-Madison, USA; \\ ${ }^{3}$ National Astronomical Observatories of China, China; ${ }^{4}$ University of Tasmania, Australia; \\ ${ }^{5}$ Centro de Radioastronomía y Astrofísica, UNAM, Morelia, Mexico; ${ }^{6}$ Columbia University, \\ USA $;{ }^{7}$ CNRS - Institut d'Astrophysique Spatiale, Université Paris-XI, Orsay, France; ${ }^{8}$ Sydney \\ Institute \\ for Astrophysics, University of Sydney, Australia; ${ }^{9}$ International Centre for Radio Astronomy \\ Research, University of Western Australia, Australia \\ E-mail: naomi.mcclure-griffiths@csiro.au
}

\begin{abstract}
As we strive to understand how galaxies evolve it is crucial that we resolve physical processes and test emerging theories in nearby systems that we can observe in great detail. Our own Galaxy, the Milky Way, and the nearby Magellanic Clouds provide unique windows into the evolution of galaxies, each with its own metallicity and star formation rate. These laboratories allow us to study with more detail than anywhere else in the Universe how galaxies acquire fresh gas to fuel their continuing star formation, how they exchange gas with the surrounding intergalactic medium, and turn warm, diffuse gas into molecular clouds and ultimately stars. The $\lambda 21-\mathrm{cm}$ line of atomic hydrogen (H I) is an excellent tracer of these physical processes. With the SKA we will finally have the combination of surface brightness sensitivity, point source sensitivity and angular resolution to transform our understanding of the evolution of gas in the Milky Way, all the way from the halo down to the formation of individual molecular clouds.
\end{abstract}

Advancing Astrophysics with the Square Kilometre Array

June 8-13, 2014

Giardini Naxos, Italy

\footnotetext{
* Speaker.
} 


\section{Introduction}

The next decades offer us the opportunity to revolutionize our understanding of how galaxies form and evolve. The current paradigm is that galaxies form at the nexus between colliding streams of cold dark matter, where new stellar systems form through bursts of star formation (SF; e.g. Vogelsberger et al. 2014). Strong winds driven by SF can force gas out of galaxies (Veilleux et al. 2005) and conversely galaxies must accrete new material to sustain their SF rates. In addition, there is mounting evidence from numerical simulations that molecular clouds form at the interfaces of colliding streams of warm atomic gas, at least under conditions similar to those in the present Milky Way. Thus, there appears to exist a continuous gas flow from extragalactic scales down to stellar scales.

The Milky Way (MW) and Magellanic System are ideal laboratories for studying the evolution of gas in galaxies. These systems have a wide range of physical conditions, including high and low interstellar heavy element abundance and SF rates. They are close, so close that the SKA will achieve spatial resolution finer than one parsec, in some cases as small as an AU. With this extreme spatial resolution, in combination with high spectral resolution, we can resolve many of the open questions about galaxy evolution: how galaxies acquire fresh gas, how they feed gas to local environments and how they turn warm, diffuse gas into cold, dense gas and ultimately form stars. In addition, the Magellanic Stream (MS), Large Magellanic Cloud (LMC), and Small Magellanic Cloud (SMC) showcase how rapidly star-forming gas driven by tidal interactions gets out of dwarf galaxies, eventually being accreted into larger systems, in this case the MW. They also show how the physical conditions of the interstellar medium (ISM) vary with heavy element abundance, gravitational potential depth, and radiation field. The MW is in many ways an archetypal spiral galaxy, and so is an important point of comparison for extragalactic studies. In addition, there is some evidence from its star formation rate and global color that the MW is undergoing one of the most important transitions in galaxy evolution, leaving its period of rapid star formation and entering the so-called "green valley", and that it will have all but extinguished its star formation in less than 5 Gyr (Mutch et al. 2011). Studying the cold gas in the MW will elucidate how star formation is quenched during this pivotal era. Thus, it is of fundamental importance to characterize the gas flow into, within, and from the Galaxy.

The $\lambda 21-\mathrm{cm}$ line is an excellent tracer of the neutral interstellar medium (ISM) in galaxies. Atomic hydrogen (H I) is found in a variety of environments, from dense clouds to the diffuse galactic halo and shows structure with size scales from kilo parsecs to a few tens of AU. Galactic $\mathrm{H}$ I spectral lines vary in width from $\sim 1 \mathrm{~km} \mathrm{~s}^{-1}$ to $\sim 60 \mathrm{~km} \mathrm{~s}^{-1}$, tracing gas with temperatures from $20 \mathrm{~K}$ to $8000 \mathrm{~K}$. The SKA will combine surface brightness sensitivity with the angular resolution provided by long baselines to deliver a MW gas survey machine. Combining SKA H I data with single-dish or auto-correlation data for the so-called "zero-spacing" will probe $\mathrm{H}$ I in the MW and Magellanic Clouds (MCs) over its full range of size scales and temperatures.

The combination of excellent $\mathrm{H}$ I surface brightness sensitivity and dense coverage of $\mathrm{H} \mathrm{I}$ absorption measurements will enable measurements of the multiphase gas flows into and out of the Milky Way disk and MCs. In combination with tracers of molecular gas and dust, such as maps made by Planck and Herschel, these $\mathrm{H}$ I data will reveal the transitions between atomic and molecular material within interstellar clouds that are crucial for star formation. Only the SKA will be able 
to capture these clouds in the MCs for the first time at sub-parsec resolution, providing important tests of the effect of metallicity and radiation field on molecular cloud formation and evolution. Over the next decade, GAIA and the Large Synoptic Survey Telescope (LSST) will provide stellar distance measurements for constructing 4D (3D plus velocity) maps of nearby nearby interstellar clouds. Moreover, the SKA will make it feasible to perform large-scale absorption surveys of the $\mathrm{OH}$ molecule. It has recently been realized that this molecule may be an ideal tracer of the socalled CO-free molecular gas, thus allowing us to investigate in detail the transformation of gas from atomic to molecular.

In this chapter we outline a number of areas where the SKA will transform our understanding of how the MW and Magellanic System work. This understanding will provide much of the physical underpinning for how large spirals and dwarf irregular galaxies evolve.

\section{The Structure and Evolution of Gas in the Milky Way and Magellanic System}

\subsection{Understanding how gas accretes onto, moves within, and is lost from a galaxy}

Galaxies are not closed systems. The evolution of the MW is significantly impacted by the two-way flow of gas and energy between the Galactic disk, halo, and intergalactic medium. We have long known that there is an extended halo of gas, both atomic and ionized, far beyond the disk of the Galaxy (e.g. Lockman 1984; Kalberla \& Dedes 2008; Reynolds 1991; Gaensler et al. 2008). In recent years we have also come to realize that the atomic portion of the halo is a highly structured and dynamic component of the Galaxy. Despite these advances we are far from understanding the origin of the gaseous halo and its interaction with the disk of the Galaxy. There are two dominant sources of structure in the halo: one is the outflow of gas from the Galactic disk, and the second is the infall of gas from extragalactic space (Putman et al. 2012). The interplay of these sources and their relative importance on the global evolution of the MW, and MW-like galaxies, is not known.

\subsubsection{Accretion}

Cosmological simulations predict that gas accretion onto galaxies is ongoing into the present epoch (e.g Joung et al. 2012). Maintaining the star formation rate measured in galaxies across cosmic time requires significant amounts of gas infall (e.g. Hopkins et al. 2008). The Milky Way is a clear example of this. With $\sim 5 \times 10^{9} \mathrm{M}_{\odot}$ of gas in the disk and a current star formation rate of $1-3 \mathrm{M}_{\odot}$ year $^{-1}$, it is clear that the Galaxy would exhaust its supply of star-forming gas in only a few Gigayears (Putman et al. 2012). The problem of providing new gas to facilitate star formation is not just limited to the current epoch. Measurements of stellar ages in disk stars show that they have formed continuously over the past $\sim 12$ Gyrs (e.g. Bensby et al. 2014) and chemical evolution models can only reproduce the abundances observed in old stars by assuming continuous accretion of low metallicity gas (e.g. Chiappini et al. 2001; Schönrich \& Binney 2009).

An outstanding source of low-metallicity star formation material for disk galaxies is gas accretion from the disruption of neighboring dwarf galaxies. The Magellanic System, created from the interaction between the SMC, LMC and the MW, provides the closest example of galaxy fueling. While the Magellanic Leading Arm is believed to be closely interacting with the MW disk, the northern tip of the MS is furthest from the MW and contains a wealth of small scale structure (Stanimirović et al. 2008). Surrounding the neutral MS is a significant pool of ionized gas, indicated 


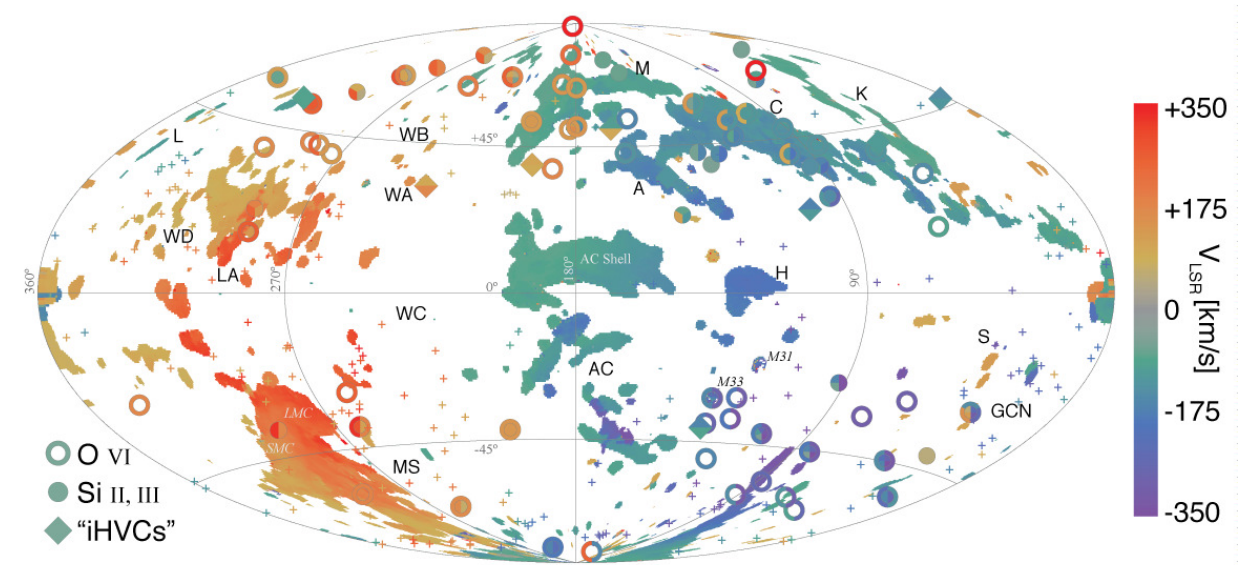

Figure 1: Distribution of high velocity clouds (HVCs) detected in H I and overlaid with measurements of low column density absorbers traced via ultraviolet metal lines (circles and diamonds). The plus symbols represent compact $\mathrm{HI}$ clouds, the distribution of which will change dramatically with SKA. The color scale indicates the local standard of rest velocity of the gas as shown in the bar at the right. From Putman, Peek \& Joung (2012).

by UV absorption lines, which may account for as much as three times the mass of the neutral gas (Fox et al. 2014). By imaging both the neutral and the ionized gas we will be able to model how the gas is being broken down, and ultimately how it is accreting onto the MW. To image the neutral gas we will need the combination of surface brightness sensitivity, spatial coverage and angular resolution provided by the SKA. Detailed H I images to a low column density limit will allow us to study the physical and thermal structure of the Magellanic System throughout the halo and probe the interaction between the Magellanic H I features and the MW disk and halo. The H I images can be compared with three-dimensional maps of the warm, ionized gas made with large-area, high kinematic resolution integral field units on 8-m optical telescopes to show the flow of warm gas relative to the disk. These studies will reveal the dynamical and thermal instability processes that are essential for feeding external material into galaxies. The SKA will also allow us to trace cold gas throughout the Stream by resolving, spatially and spectrally, cold cores, and allowing us to search for absorption through the Stream. Finally, these studies will help us to probe the physical properties of the halo itself, using the Stream clouds as test particles in the halo.

Another potential source of accreted gas is the reservoir of $\mathrm{H}$ I in the form of high velocity clouds (HVCs, see Figure 1); however, these clouds fall about an order of magnitude short of what is needed (Putman et al. 2012). Some of the missing mass is in the form of ionized gas (e.g. Lehner et al. 2012; Fox et al. 2014) and another, undetermined, small fraction may be in the form of small, dense clumps of optically thick material. While the ionized gas is best traced by UV and optical absorption lines, gas at $T<8000 \mathrm{~K}$ is well-traced by $\mathrm{H} \mathrm{I}$. Our understanding of the link between the $\mathrm{H}$ I gas detected in emission and warm gas detected in ultraviolet absorption will change dramatically with the SKA. While high velocity $\mathrm{H}$ I gas detected in emission has been estimated to cover $\sim 35 \%$ of the sky, high velocity gas detected in absorption using metal lines is found to cover on the order of $80 \%$ of the sky. This indicates there is a large reservoir of gas in the Galaxy's halo with column densities of $\mathrm{N}(\mathrm{H} \mathrm{I})<10^{18} \mathrm{~cm}^{-2}$ that remains to be detected in $\mathrm{H} \mathrm{I}$ 
emission (e.g. Lehner et al. 2012). This is consistent with what has been found in other galaxy halos (e.g. Werk et al. 2014). Very deep integrations of $\mathrm{H}$ I and spatial stacking have detected some of this diffuse $\left(\mathrm{N}(\mathrm{H} \mathrm{I}) \sim 2 \times 10^{18} \mathrm{~cm}^{-2}\right.$ ) medium as HVC envelopes with FWHM $\sim 60 \mathrm{~km} \mathrm{~s}^{-1}$ at the interface with the hot halo gas (Nigra et al. 2012). This diffuse low column density material could be important for cloud lifetimes and overall accretion onto the Milky Way. To detect this gas we require a combination of extremely high surface brightness sensitivity and "zero-spacing" observations for H I mapping of all angular scales. Combining these maps with sensitive H I absorption observations, the SKA will be able to detect and map both the cold and warm gas reservoirs of Milky Way star formation fuel.

We also know that cool $(T<1000 \mathrm{~K})$ gas exists in the HVC population. In some cases, $\mathrm{H}$ I cores have been found to be associated with optical and UV absorption line systems when synthesis observations are completed (Ben Bekhti et al. 2009). Kalberla \& Haud (2006) find that $24 \%$ of HVC sight-lines show narrow-line width $\left(\Delta v \sim 7 \mathrm{~km} \mathrm{~s}^{-1}\right)$ components consistent with multiphase structure in the condensed halo. Evidence from higher resolution observations suggests that beam dilution hides some of this cool gas (Moss 2014). Furthermore, data from FUSE show $\mathrm{H}_{2}$ absorption in more than a dozen high and intermediate velocity clouds (Richter et al. 2001). Unfortunately, most of what we know about the multiphase $\mathrm{H}$ I in the MW halo is derived from single dish observations, which lack the spatial resolution to clearly detect small, cold clumps of $\mathrm{H}$ I. We know very little about the temperature, distribution or quantity of cool $\mathrm{H}$ I in the halo. At present there are only two measurements of $\mathrm{H}$ I gas excitation, or spin, temperature in an HVC (Wakker et al. 1991; Matthews et al. 2009). Absorption line measurements with the SKA, with its resolution and sensitivity, will comprehensively determine the amount of condensed gas in the halo enabling direct comparisons with theoretical and numerical models (e.g. Wolfire et al. 1995, Joung et al. 2012). Furthermore, estimates of the mass of $\mathrm{H} \mathrm{I}$ in HVCs rely on the assumption of optically thin gas when calculating column densities (Putman et al. 2002; Moss et al. 2013), leading to underestimates of the total HVC mass in the halo. Using NVSS continuum source counts (Condon et al. 1998) we estimate that with the SKA we will measure hundreds of absorption components through HVCs, giving estimates of H I spin temperatures and opacity in HVCs and estimates of the fraction of gas "missed" by optical depth assumptions.

\subsubsection{Outflow}

A significant fraction of the gas in the Galactic halo may also be attributed to the outflow of structures formed in the disk, but extending into the halo. Large-scale H I "chimneys" (e.g. McClure-Griffiths et al. 2006; Pidopryhora et al. 2007) supply hot, metal-enriched gas to the Galactic halo via the "Galactic Fountain" (Shapiro \& Field 1976; Bregman 1980) and also may be a dominant source of multi-phase gas structure for the lower halo. Dense, cool condensations from expelled gas (e.g. de Avillez 2000) and the tops of H I chimneys that break at $z$-heights of $\sim 1$ kpc (e.g. Mac Low et al. 1989) may populate the halo. Deriving the destruction timescales and physical properties (temperature, density) of multiphase fountain gas will inform our estimates of the survival times and journey histories for cold halo cloud populations. High sensitivity SKA observations of $\mathrm{H} \mathrm{I}$ in emission and absorption, together with observations of molecular and ionized gas tracers, will reveal the multiphase structure of gas outflowing from the disk. Comparison of 
multiphase gas properties with heating and cooling models (e.g. Wolfire et al. 1995) will determine the pressure and thermal structure of the lower halo of the MW.

Galactic fountain gas may even play an important role in seeding the halo to trigger gas accretion from so-called hot-mode accretion. Fraternali et al. (2013) have suggested that high-metallicity expelled gas mixes efficiently with hot halo gas and triggers the cooling of such gas in the lower halo. This cooled gas is observed as absorption features (e.g. Lehner et al. 2012) and can efficiently accrete onto the disk. Fraternali et al. estimate that supernova-driven fountain cooling produces a net gas accretion onto the disk at a rate of a few $\mathrm{M}_{\odot} \mathrm{yr}^{-1}$ and speculate that this mechanism explains how the hot mode of cosmological accretion feeds star formation in galactic disks. This intriguing suggestion can be tested by combining SKA H I observations with UV/optical absorption line measurements. A sensitive SKA survey of $\mathrm{H}$ I emission in the lower halo of the Milky Way will reveal the predicted low column density $\left(<10^{19} \mathrm{~cm}^{-2}\right)$ tails of Galactic fountain material. These images, combined with $\mathrm{H}$ I absorption will directly associate cool and warm $\mathrm{H} \mathrm{I}$, measure temperatures, and trace its origins to the disk.

\subsubsection{The SKA and Gas Tomography}

The development of the SKA is happening in tandem with an ongoing explosion of stellar data. The gaseous properties can be linked to the dust and distance measurements made using detections of stars in the halo of the MW and LMC from future large optical surveys, such as Pan-STARRS and LSST. By measuring the colors of stars with enormous photometric surveys we are able to build 3D tomographic maps of the dust distribution in the Galaxy (Green et al. 2014; Schlafly et al. 2014). In many circumstances, especially in low-density environments, H I and dust trace the same underlying distribution of material. For example, by looking at absorption lines toward stars, there have been some measurements of distances to high velocity gas (e.g. Thom et al. 2008), and from those estimates of accretion rates onto the Galaxy (Putman et al. 2012). Thus, if we are able to combine our kinetic information from a high sensitivity SKA Galactic H I survey with the tomographic information from stellar surveys we will be able to produce truly 4D (3D plus velocity) kinetic tomography maps of gas flow to and from the Milky Way, revealing how feedback works in disk galaxies.

Within our Galaxy's disk, structures are formed through the collapse of diffuse material. By combining $\mathrm{H}$ I kinematics with information on the distance of gas, observations of $\mathrm{H}$ I represent a direct measurement of one of the most fundamental questions in all astronomy, "where did this come from"? To understand formation using observations of diffuse gas we look to the continuity equation, which relates the rate of change of density $(\delta \rho / \delta t)$ to the spatial and kinematic state of the fluid. The position-position-velocity (or hyper-spectral) data provided by radio surveys is clearly incomplete for this task - we do not have access to distance information. This leads to highly model-dependent descriptions of the state of the Galaxy. As an example, maps of the H I surface density of the disk must rely on assumptions of a flat rotation curve to extrapolate into the third spatial dimension (e.g. Levine et al. 2006). Even with these assumptions, we lose information about flows of the gas beyond simple rotation, and thus are blind to the more complex dynamics at play. The high angular resolution $\mathrm{H}$ I data cubes that we will obtain with the SKA can be used in conjunction with deep photometric stellar surveys to finally enable 4D kinetic tomography maps of gas flow within the MW. Combining high angular resolution H I data, which provides the 
information we need to isolate individual $\mathrm{H}$ I structures, with next-generation photometric surveys of tens of billions of stars which give us precision reddening information, will allow us to determine the distance to many Galactic $\mathrm{H}$ I structures enabling reconstruction of the detailed structure of the MW disk. With the full kinematic information, vertical oscillations and corrugations of the MW disk will be related for the first time to small-scale dynamical effects of spiral arms, giant molecular clouds, and/or sites of stellar energy injection.

\subsection{Tracing the Life-cycle of Hydrogen from Diffuse to Molecular}

The current paradigm is that gas in the MW is continually flowing between its various thermal states. The flow changes gas from its warm $T \sim 10^{4} \mathrm{~K}$ state, through an intermediate unstable phase, to its cold atomic form, with temperatures ranging from $\sim 20 \mathrm{~K}$ to $500 \mathrm{~K}$. How exactly atomic gas transitions into denser, colder clouds in which the hydrogen is mostly in molecular form and in which stars form, is still not understood.

The corollary of this hydrogen life-cycle is that the formation of stars ultimately destroys the molecular clouds, and a large fraction of the gas is returned to either ionized or atomic form. Part of understanding how a galaxy evolves is understanding how much hydrogen exists in its various states and the flow rates between states and scales. The SKA is the only instrument capable of providing direct and statistically significant measurements ( $\sim 2 \times 10^{5}$ sources) of neutral gas states over the full temperature range from 20 to $10^{4} \mathrm{~K}$, while simultaneously probing diverse interstellar environments and spatial scales from AU to kiloparsecs.

\subsubsection{Distribution of Mass and Temperature in the Cool, Unstable and Warm Atomic Medium}

Interstellar gas is subject to strong radiative heating and cooling processes which, within certain density or temperature ranges, may cause a gas parcel to become depressurized when it is compressed (Field 1965; Field et al. 1969; Wolfire et al. 1995) leading to the so-called thermal instability and a runaway compression which lasts until the parcel exits the unstable range. This process tends to segregate the atomic gas into a warm/diffuse phase called the warm neutral medium (WNM) and a cold/dense phase called the cold neutral medium (CNM). The classical picture arising from this is that the medium should exist in two or three main stable phases (Field et al. 1969; McKee \& Ostriker 1977) with very little gas in the thermally unstable range. However, both observational (e.g. Dickey et al. 1977; Kalberla et al. 1985; Heiles \& Troland 2003) and numerical (e.g. Vázquez-Semadeni et al. 2000; Gazol et al. 2001, 2005; Audit \& Hennebelle 2005; Hill et al. 2012) studies have suggested the presence of significant amounts of unstable gas. The existence of this unstable gas could be understood theoretically as a consequence of several physical processes. Small-scale changes in pressure caused by spiral arms or the distance from the Galactic mid-plane can alter the temperature range where stable gas is observed. Alternatively, the mixing action of turbulence, which causes the gas to flow from one phase to another, can produce gas in the traditionally unstable temperature range. However, the predicted fraction of thermally unstable gas varies hugely across numerical models. Direct comparison with observations over focused interstellar conditions are highly required to constrain the above physical processes and timescales on which they operate. 
The mass fraction in each of the hydrogen phase regimes, including the unstable regime, is driven by the heating and cooling processes and therefore is highly sensitive to parameters such as metallicity, the interstellar radiation field and the strength of the turbulence (Wolfire et al. 2003). Surprisingly, even the fractions of the total H I mass in the CNM and WNM for most regions of the Galaxy are poorly known and almost nothing is known about how they vary with Galactic position. Our knowledge of the unstable gas fraction is even worse and the flow rates between stable and unstable phases are virtually unknown.

With the SKA we will achieve statistically meaningful measurements of the distribution of mass and temperature in the CNM and WNM, as well as the unstable phase. The SKA, with a deep survey of the Galactic plane and lower halo, will provide those measurements through detections of $\mathrm{H}$ I absorption, probing spiral arms, inter-arm regions and distance from the mid-plane. For the first time we will be able to measure gas spin temperature,distribution functions at all Galactocentric radii, as well as in and above the plane, and close to some major Giant Molecular Clouds (GMCs) with unprecedented spatial coverage of absorption sources. With this we can test the theoretical predictions on how the spin temperature varies with metallicity, interstellar radiation field and pressure for the first time.

\subsubsection{Molecular cloud formation}

Recent numerical studies suggest that cold, dense clouds of atomic hydrogen form by a phase transition from the WNM to the CNM induced by large-scale compressions in the warm gas (Ballesteros-Paredes et al. 1999; Audit \& Hennebelle 2005; Heitsch et al. 2005, 2006; VázquezSemadeni et al. 2006). Such compressions may be a consequence of the general turbulence in the WNM or by large-scale instabilities that drive converging flows in this medium. Cold clouds formed by this mechanism can eventually develop column densities large enough for molecules to form, and ultimately form stars. Simulations of the formation of GMCs are rich and show that GMC formation can happen via many different avenues. One possibility is that cold clouds develop gravitational instabilities, which lead to contraction. Thus, molecular gas and stars may be the result of gravitational contraction starting in the atomic phase (e.g Vázquez-Semadeni et al. 2007; Heitsch et al. 2008; Heitsch \& Hartmann 2008; Heitsch \& Putman 2009; Banerjee et al. 2009) which, along the way, also produces complicated filamentary structures (Gomez \& VazquezSemadeni 2013). As the gas flows from the WNM into the dense molecular structures, transitional regions that are mostly cold atomic in their outermost parts, then CO-free molecular, and finally CO-bearing molecular in their innermost parts are produced (Smith et al. 2014; Heiner et al. 2014). Alternatively, GMCs may develop from an agglomeration of small cold clouds, collected together by the sweeping action of spiral arms (Dobbs et al. 2012) or through the compressive action of superbubbles whose walls collect and compress small H I clouds (Ntormousi et al. 2011; Clark et al. 2012).

The detail apparent in the simulations is not yet matched by observational data. While undoubtedly most of the simulated physical processes are involved in forming molecular clouds, we do not have the observational handles to determine which processes dominate in different environments. Observing the flow pattern of cold atomic gas is difficult because of the confusion in the atomic gas along lines of sight inside our Galaxy. Dominated by turbulence and the overlay of structures, the spectral structure of Galactic $\mathrm{H}$ I line profiles is usually wider than $7 \mathrm{~km} \mathrm{~s}^{-1}$. By 
comparison, typical molecular line widths, although supersonic, are mostly on the order of $\sim 1$ $\mathrm{km} \mathrm{s}^{-1}$. This mismatch of spectral characteristics is the main reason why the atomic to molecular transition in ISM has not been mapped systematically, in spite of the existence of both large scale H I maps and large scale CO maps for more than three decades. The observational challenge lies in objectively associating flux in the $\mathrm{H}$ I line with a specific molecular structure, which is orders of magnitude smaller both in spatial extent and turbulence magnitude. SKA H I absorption measurements, combined with high surface brightness sensitivity data at high Galactic latitude and sub-arcminute resolution will generate a substantial catalogue of isolated $\mathrm{H}$ I clouds with simple Gaussian line profiles. These clouds can be observed with matching angular ( $\sim 20$ ") and velocity $\left(0.3 \mathrm{~km} \mathrm{~s}^{-1}\right)$ resolutions in molecular tracers, such as $\mathrm{OH}, \mathrm{CO}$ and $\mathrm{HCO}^{+}$. The matching of molecules and atoms will enable quantitative analysis of important atomic to molecular transitions in many well-defined systems.

The SKA will finally give us the ability to observe the flow of cold $\mathrm{H}$ I onto molecular clouds and the mixture of phases within molecular clouds. One important tool in studies of cold gas flow is the wide-spread effect of H I Self-Absorption (HISA), which is observed when cold foreground $\mathrm{H}$ I absorbs background H I emission at similar velocities. Many of these features are completely without molecular counterparts (Gibson et al. 2005). Within molecular clouds, the collision with $\mathrm{H}_{2}$ produces a much colder and generally narrower feature often called HI Narrow Self-Absorption (HINSA; Li \& Goldsmith 2003). HISA and HINSA allow us to map the structure of cold gas, but because the temperature of the background emission is unknown we cannot know the temperature of the cold gas. By contrast, $\mathrm{H}$ I absorption toward continuum sources gives us the spin temperature of the gas, $T_{s}$, but not the spatial structure. Using the SKA we will finally be able to use a combination of HISA and H I continuum absorption to understand the spatial and thermal structure of cold H I. An SKA survey of the Milky Way will enable sensitive absorption studies toward background quasars giving opacity measurements with source density of $>10 \mathrm{deg}^{2}$. This survey will provide multiple absorption spectra across any given HISA feature, effectively providing a temperature gauge to calibrate the HISA optical depth. This powerful technique will provide accurate measurements of gas temperatures and column densities around and within molecular clouds. The SKA data will be combined with detailed studies of individual molecular clouds from ALMA for a complete picture of the flow of cold gas into and through molecular clouds.

The SKA will also enable essential complementary surveys of the hydroxyl $(\mathrm{OH})$ molecule, whose emission is very weak, despite its probable ubiquity and large observed column densities. Recent evidence that a large fraction of molecular gas exists in the so-called "dark molecular gas" (or CO-free) form (Grenier et al. 2005; Planck Collaboration et al. 2011; Langer et al. 2010) amplifies the need to find other tracers of cold $\mathrm{H}_{2}$. In models of photo-dissociation regions (PDRs), $\mathrm{OH}$ can form quickly in terms of extinction after $\mathrm{H}_{2}$ becomes self-shielded (van Dishoeck \& Black 1988). A series of charge exchange reactions facilitated by cosmic rays produces OH. Due to its low excitation temperature, however, large scale maps of $\mathrm{OH}$ emission has not been available. A systematic and large scale $\mathrm{OH}$ absorption survey, possible with the sensitivity afforded by the SKA, will quantify the amount of this potentially most abundant diatomic molecule after $\mathrm{H}_{2}$ and lead to a Milky Way-wide measurement of dark molecular gas. The column densities and temperatures of $\mathrm{OH}$ will be the cornerstone to build models of $\mathrm{H} \mathrm{I-} \mathrm{H}_{2}$ transition. 


\subsubsection{The multi-scale SKA meets the multi-scale ISM}

Turbulence is a concept often applied to random variations in the density and velocity fields of the ISM. The term implies a stochastic process that transfers kinetic energy from larger to smaller scales in a cascade similar to the well known Kolmogorov process (Kolmogorov 1941, 1962, reviewed by Elmegreen and Scalo 2004, Falceta-Gonçalves et al. 2014), although only a few studies exist about the nature of the cascade in the strongly compressible case (e.g., Kritsuk et al. 2007). Interstellar turbulence affects many different kinds of observations, from spectral line cubes to pulsar scintillation. It is present in all phases of the ISM, yet its exact energy sources and sinks are still not identified. Interstellar turbulence can be measured quantitatively either through the structure function or power spectrum (Rickett 1977; Dickman \& Kleiner 1985; Lazarian \& Pogosyan 2000). The 21-cm line can be used in emission or absorption to trace turbulence in both the WNM and $\mathrm{CNM}$ over a broad range of scales. The SKA will connect the $\mathrm{H}$ I with turbulence in the warm ionized medium (WIM) on large-scales as traced by pulsars (Armstrong et al. 1995) and polarization observations of the magneto-ionic medium (Gaensler et al. 2011). On small scales the SKA will connect the CNM with turbulence in molecular clouds, where fluctuations in the density and velocity fields are generally thought to shape the processes of gravitational collapse and star formation (Larson 1981, Qian et al. 2012; see also the review by McKee and Ostriker 2007), although recently it has been proposed that, instead, these fluctuations are the result of global gravitational contraction in the clouds rather than the other way around (Ballesteros-Paredes et al. 2011).

Spectral line cubes of the 21-cm H I emission brightness over a large area with a small beam are used to characterize the turbulence spectrum over a range of scales, typically from $\sim 100 \mathrm{pc}$ to $\sim 0.1$ pc (Chepurnov et al. 2010; Pingel et al. 2013). The SKA will provide the high sensitivity and large numbers of independent emission spectra needed to compute the spatial power spectrum in the Galactic ISM down to AU scales and with precision and statistical confidence better than in previous studies (Crovisier \& Dickey 1983; Green 1993; Dickey et al. 2001). In the Magellanic System, the sensitivity of a deep SKA survey will give a large improvement over the best existing data (Stanimirović \& Lazarian 2001; Elmegreen et al. 2001; Muller et al. 2003). In nearby Galactic $\mathrm{H}$ I clouds, moments of the spectral line cubes give the velocity and density structure functions that can connect the turbulence spectrum in the $\mathrm{H}$ I to the very well known spectrum measured for the WIM (Armstrong et al. 1995). It will be particularly important to test whether the neutral medium has structure similar to the extreme scattering events (ESE) in the ionized gas (Fiedler et al. 1987; Walker \& Wardle 1998), and to match structures in the H I with scattering screens deduced from pulsar scintillation (Bhat \& Gupta 2002). Additional statistical measures such as those proposed by Burkhart et al. (2010) will probe deeper into the fluctuations of the ISM.

In the CNM, variations in the optical depth and line centre velocities among $\mathrm{H}$ I absorption spectra toward nearby background sources will provide a way of tracing turbulence independently of the H I emission that is dominated by WNM. The SKA will provide orders of magnitude more absorption spectra toward compact continuum sources, making it possible to use these data to map the variations in absorption on a wide range of scales. This is particularly important to connect with the "tiny scale structure" (Heiles 1997; Deshpande et al. 2000; Braun \& Kanekar 2005) that is seen in similar variations of the absorption, particularly with VLBI (Roy et al. 2012). It will also be critical to link the turbulence in the CNM to that seen in molecular clouds using CO and 
other molecular line tracers (Falgarone et al. 2009; Krčo et al. 2008; Li \& Goldsmith 2012) using ALMA. Whether or not the turbulence in molecular clouds is "frozen-in" with the gas when it makes the transition from CNM atomic to molecular is an important question for understanding star formation. The multi-scale physics of the mass and energy cascade from large galactic scales down to the small scales on which molecular clouds shape the ISM is a challenge for our understanding of galaxy evolution. The SKA, with its inherent spatial dynamic range, is ideally designed to probe the multi-scale physics of the ISM.

\subsection{Taking ISM evolution to The Magellanic Clouds}

The MCs, including the SMC and LMC, offer a nearby example of a low-metallicity ( $Z \sim$ $0.2 \mathrm{Z}_{\odot}$ and 0.5, respectively; Dufour 1975, Olszewski 1996) environment with interstellar conditions that sharply contrast with what we find in the MW. Given their close proximity $(\sim 50-60 \mathrm{kpc}$ Westerlund 1997), these are the only external galaxies where we can study the atomic and molecular content- from dense star-forming regions to diffuse accretion streams- at high (pc-scale) spatial resolution. In addition, the intense interstellar UV radiation field of the SMC and the LMC, 4-10 times higher than that in the Solar neighborhood (Azzopardi et al. 1988), implies that heating and cooling rates, dust-to-gas ratios, and chemical abundances in the MCs represent the closest local examples of less-evolved systems common at high redshift.

\subsubsection{Interstellar environment and the fraction of cold gas}

The census of cold gas and its conversion into stars over cosmic time is one of several key parameters associated with galaxy evolution; however little is known about the cold atomic gas even in very nearby galaxies. As the "demography" of cold gas and the phase mix are largely driven by the heating and cooling processes - whose rates vary with metallicity, dust-to-gas ratio, and the strength of the interstellar radiation field - significant variations of the CNM/WNM properties and abundances are expected from a theoretical point of view (Wolfire et al. 2003). The reality is that, in our home neighborhood only $29 \mathrm{H} \mathrm{I}$ absorption measurements exist for the cold gas in the SMC (Dickey et al. 1994, 2000), the nearest metal-poor galaxy. Similarly, only a handful of H I absorption spectra exists for the LMC (Marx et al. 1997), M31 (Dickey \& Brinks 1993), and M33 (Dickey \& Brinks 1993; Braun 1997). The only recent attempt to study properties of cold gas in a lower-metallicity environment offered by the outer radii of the Milky Way resulted in a highly puzzling result. Dickey et al. (2009) suggest that the spin temperature of the CNM, and the CNM fraction, stay constant with Galactocentric radius. At a Galactocentric radius of $25 \mathrm{kpc}$, where the supernova rate, metallicity and interstellar radiation field are significantly lower than in the inner Galaxy, the temperature of cold gas, contrary to all theoretical predictions, is not different from what is found close to the Galactic centre (Strasser et al. 2007). The SKA will allow us to extend this test to lower metallicities within the LMC and SMC.

The SKA will revolutionize our understanding of the cold neutral gas in nearby galaxies, starting with the MCs. With 25 background radio continuum sources $(S>20 \mathrm{mJy})$ per square degree (Condon et al. 1998), $\sim 2000 \mathrm{H}$ I absorption spectra with $\sigma_{\tau}<10^{-2}$ will be obtained towards the MCs. This will allow us to measure the properties of the CNM in the Clouds and the mixture of warm and cool atomic gas, as well as their respective spatial distributions. A comparison 
of the CNM/WNM statistics between the Milky Way and the MCs will show the variation of the heating and cooling rates with metallicity and how these processes affect the star formation rate.

\subsubsection{Formation of $\mathrm{H}_{2}$ in galaxies}

Observational studies of galaxies (Kennicutt 1998; Bigiel et al. 2008; Schruba et al. 2011) show that the surface density of the star formation rate scales linearly with the surface density of molecular gas. This suggests that stars form in molecular clouds with a relatively similar efficiency and therefore the ability to form $\mathrm{H}_{2}$ controls the evolution of individual galaxies. Another interesting observational result that came to light over the past few years is that the surface density of atomic gas $\left(\Sigma_{H I}\right)$ on kpc-scales rarely exceeds $\sim 10 \mathrm{M}_{\odot} \mathrm{pc}^{-2}$ (e.g Bigiel et al. 2008). This saturation of $\Sigma_{H I}$ provides important pointers for the physical conditions required to form molecular gas out of the atomic medium. For example, Krumholz et al. (2009) showed that in the case of equilibrium $\mathrm{H}_{2}$ formation, a certain amount of $\mathrm{H}$ I surface density is required to shield $\mathrm{H}_{2}$ against photodissociation, and this $\mathrm{H}$ I shielding surface density depends primarily on metallicity.

Lee et al. (2012) found a relatively uniform H I surface density of 6-8 $\mathrm{M}_{\odot} \mathrm{pc}^{-2}$ for several dark and star-forming regions in the Perseus molecular cloud in the MW and showed that $\Sigma_{H I}$ and the $\mathrm{H}_{2}$ fraction agree well with the equilibrium model predictions. However, the key test for understanding $\mathrm{H}_{2}$ formation across galaxies requires observational probes of interstellar environments different from those we find in the MW. Extragalactic observations in the pre-SKA era cannot resolve individual molecular clouds. Only SKA observations of the MCs will provide crucial tests for the $\mathrm{H}$ I surface density and $\mathrm{H}_{2}$ fraction in low metallicity and high-interstellar radiation field environments on pc-scales required to test both equilibrium and non-equilibrium (e.g. Glover \& Mac Low 2007) models for $\mathrm{H}_{2}$ formation and molecular cloud evolution.

\subsubsection{The warm neutral medium and excitation of $\mathrm{H}$ I}

The WNM is one of the least understood phases of the ISM. To constrain theoretical and numerical models of the ISM and its evolution over cosmic time, temperature distributions over the full temperature range from $\sim 20$ to $\sim 10^{4} \mathrm{~K}$ are essential. This requires extremely highsensitivity observations due to the very low optical depth of the WNM, $\tau<10^{-3}$. Only two direct measurements of WNM spin temperature exist for the MW so far (Carilli et al. 1998; Dwarakanath et al. 2002). Using the upgraded Very Large Array, Murray et al. (2014) detected statistically the presence of a widespread WNM population with $T_{s}=7200 \mathrm{~K}$. This study was possible due to very deep integrations, detection and modeling of the CNM lines, and then stacking of the spectral residuals. This study demonstrates, for the first time, that the non-collisional excitation of $\mathrm{H} \mathrm{I}$ is significant even at high Galactic latitudes. As Ly $\alpha$ scattering is the most likely candidate for additional excitation of $\mathrm{H}$ I, the Murray et al. results show that the fraction of Ly $\alpha$ photons, and/or the photon propagation through the ISM, are likely more complicated than what is currently assumed. For example, both a theoretical study by Liszt (2001) and recent numerical simulations by Kim et al. (2014) assume a uniform flux of Ly $\alpha$ photons throughout the ISM and result is the expected $T_{s} \lesssim 4000 \mathrm{~K}$. Clearly, this discrepancy between observations and theory needs to be understood.

The high sensitivity enabled by the SKA will allow direct measurements of WNM spin temperature and its spatial variations for the first time. As extremely high sensitivity is needed, the 
MCs are perfect candidates for obtaining deep H I absorption spectra $\left(\sigma_{\tau}=10^{-4}\right.$ per $1 \mathrm{~km} \mathrm{~s}^{-1}$ velocity channels) to provide a statistically significant sample of the WNM. With thousands of $\mathrm{H} \mathrm{I}$ spectra we will also be able to stack selectively to probe different interstellar environments (e.g. close to major star forming regions vs galaxy outskirts).

Finally, while the H I content of the MCs has been extensively studied alone and in synergy with other multi-wavelength observations (e.g. Kim et al. 1998; Stanimirović et al. 1999; Leroy et al. 2007; Meixner et al. 2013), the SKA will provide necessary sensitivity and angular resolution to match $\mathrm{H}$ I images with the resolution of Spitzer, Herschel (18" and $12^{\prime \prime}$, respectively), and ALMA images.

\subsection{The Milky Way as a Foreground for Cosmology}

To properly measure the magnitudes and colors of populations of extragalactic objects, a correction for the extinction and reddening caused by dust in the MW is crucial. There are classically two ways of mapping this correction across the high Galactic latitude sky. The first is to measure the far-infrared (IR) emission from dust particles and make an assumption about the ratio of this emission to reddening and extinction. This method has been implemented by Schlegel et al. (1998, SFD). The second is to assume dust and neutral gas are well mixed, and that neutral gas dominates the column density, so that the $\mathrm{H}$ I column density can be used as a proxy for extinction (Burstein \& Heiles 1978). It has recently been shown that known biases in the SFD dust map affect large-scale structure observations to the point of significantly biasing estimation of key cosmological parameters (Huterer et al. 2013), such as the evolution of the equation of state of dark matter $(d w / d t)$ or cosmic non-Gaussianity. Thus, we require higher precision, less biased dust maps than are currently available to make best use of planned cosmological studies of large-scale structure. It has also recently been shown that better dust maps can be constructed by combining far-IR-based methods and H I-based methods (Peek 2013). Thus, a high-resolution H I column density map of the high-latitude sky is a crucial product of the SKA. At present it is not known whether the CNM and WNM phases of the ISM have significantly different ratios of reddening to integrated $\mathrm{H}$ I line intensity, but it is likely, as differing gas volume densities modify grain depletion (Wakker \& Mathis 2000). If this is the case, it is crucial that a high latitude survey not only have high angular resolution and sensitivity, but also high spectral resolution to get independent estimates of CNM (narrow) and WNM (broad) columns for each line of sight.

\section{Goals for SKA1}

Significant advances in our understanding of the evolution of the H I in the MW and Magellanic System from kiloparsec to AU scales can be achieved through two surveys, both with high spectral and spatial resolution. One survey will be a shallow, high spectral resolution, all-sky survey of Galactic H I, which could be carried out commensally with an all-sky continuum survey covering $1420 \mathrm{MHz}$. The second will target the Galactic Plane and Magellanic System with high sensitivity, spectral resolution and angular resolution. The two surveys are described below. Critical to both surveys are the requirements for: a) high velocity resolution on the order of $\sim 0.3$ $\mathrm{km} \mathrm{s}^{-1}$ to resolve narrow spectral lines of $\Delta v \sim 2 \mathrm{~km} \mathrm{~s}^{-1}$, b) excellent surface brightness sensitivity for imaging of diffuse $\mathrm{H}$ I emission, c) capacity to recover "zero-spacing" flux by combining 

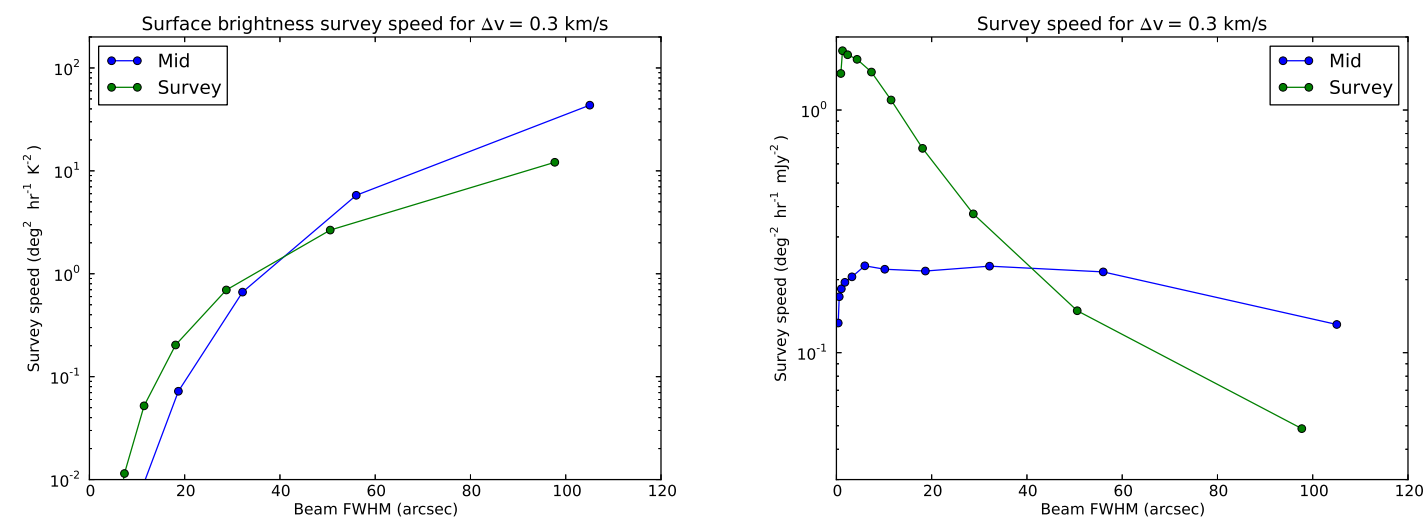

Figure 2: Comparison of $\mathrm{H}$ I line survey speeds for $0.3 \mathrm{~km} \mathrm{~s}^{-1}$ channels on SKA1-MID and SKA1-SUR for left): surface brightness limited survey and right): flux limited survey. These are derived from simulations using the latest SKA1-MID and SKA1-SUR baseline distributions and assuming uniform weighting (Popping et al. 2014) and agree with the Braun (2014) SKA performance document.

interferometric and single dish data and d) significant long baseline coverage to enable accurate $\mathrm{H} \mathrm{I}$ absorption measurements.

Brightness temperature limited survey speed as a function of beam size for both SKA1-SUR and SKA1-MID is shown in Figure 2 (left) assuming a channel width of $0.3 \mathrm{~km} \mathrm{~s}^{-1}$. Clearly for heavily tapered data at angular resolutions of $>40$ " the survey speed for SKA1-MID exceeds that of SKA1-SUR by up to a factor of a few. However, at the angular scales of interest for Galactic H I $(<30$ ") the surface brightness limited survey speed for SKA1-SUR is about a factor of 1.5 times that for SKA1-MID. For measurements of H I absorption, the flux sensitivity at a spectral resolution of $0.3 \mathrm{~km} \mathrm{~s}^{-1}$ at the natural resolution of the array, $\sim 5$ ", is the important quantity. In Figure 2 (right) we see that for $\sim 5$ " resolution the survey speed for SKA1-SUR is more than a factor of 10 faster than SKA1-MID. To achieve the simultaneous goals of Galactic H I emission and absorption SKA1-SUR is clearly the optimum instrument. Assuming SKA1-SUR we estimate the expected brightness temperature sensitivity, $\sigma_{T}$, with an angular resolution of 30 " and flux density sensitivity, $\sigma_{S}$, at 5 " per $0.3 \mathrm{~km} \mathrm{~s}^{-1}$ channel for three nominal integration times of $200 \mathrm{hr}, 50 \mathrm{hr}$ and $8 \mathrm{hr}$ (dwell time) as given in Table 1.

Table 1: Sensitivities for various dwell times for SKA1-SUR

\begin{tabular}{lccc}
\hline \hline Dwell time & $\sigma_{T}(30 ")$ & $\sigma_{S}\left(5^{\prime \prime}\right)$ & Absorption sources $\left(\mathrm{deg}^{-2}\right)$ \\
\hline $200 \mathrm{hr}$ & $0.3 \mathrm{~K}$ & $0.2 \mathrm{mJy}$ & 25 \\
$50 \mathrm{hr}$ & $0.7 \mathrm{~K}$ & $0.5 \mathrm{mJy}$ & 14 \\
$8 \mathrm{hr}$ & $1.7 \mathrm{~K}$ & $1.2 \mathrm{mJy}$ & 8 \\
\hline
\end{tabular}

\subsection{All-sky Survey of $\mathrm{H}$ I Emission and Absorption with SKA1}

Using SKA1 we will study the interplay of warm and cold atomic gas in the disk and halo by conducting a two-year all-sky survey of $\mathrm{H}$ I emission and absorption with SKA1-SUR. We estimate the observing times required to achieve necessary sensitivities for the two components of 
this survey on SKA1-SUR. Imaging of H I emission will probe the diffuse circumgalactic MW and MS, enabling many of the scientific goals outlined above. A 2-year survey ( 8 hours per pointing) will give a brightness temperature limit over the whole sky at $30^{\prime \prime}$ of about $1.7 \mathrm{~K}$ per $0.3 \mathrm{~km} \mathrm{~s}^{-1}$ channel, which is comparable to the most sensitive Galactic plane interferometric surveys but covers the whole sky, including the disk-halo interface and the Magellanic System, at a factor of six better angular resolution. This survey will probe the structure of the circumgalactic gas, the MS and gas at the interface between the disk and halo. Diffuse emission requires significant numbers of short baselines for surface brightness sensitivity and recovery of extended emission. Furthermore, to understand the nature of the diffuse $\mathrm{H} \mathrm{I}$, which fully covers the sky we will need to include "zero-spacing" information, as discussed in $\$ 3.3$ below.

The transformative aspect of the SKA1 all-sky survey will be the most extensive study of H I absorption in the Galaxy and Magellanic System ever conducted, probing the physical properties of gas throughout the Galactic halo. A 2-year all-sky SKA1-SUR survey will give $\sigma_{S}=1.2 \mathrm{mJy}$ in 0.3 $\mathrm{km} \mathrm{s}^{-1}$ spectral channels. For $\mathrm{H}$ I absorption, the flux density sensitivity converts to optical depth sensitivity of $\sigma_{\tau}=\sigma_{S} / S_{b k g}$ for a given value of the background source flux density, $S_{b k g}$. Using Condon et al. (1998) continuum source counts we estimate the number of optical depth detections of $\sigma_{\tau}<0.05$ per square degree in Figure 3. The density of sources is also given in column 4 of Table 3. We expect to obtain precision measurements of $\sigma_{\tau}<0.05$ towards $\sim 8$ sources deg $^{-2}$ or a total $\sim 2 \times 10^{5}$ sources.

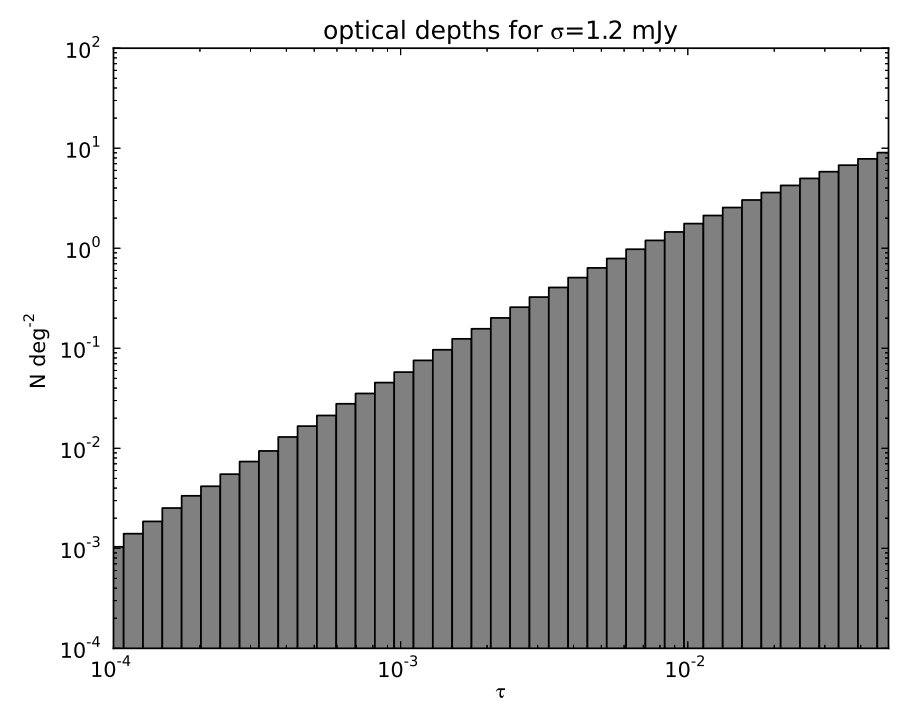

Figure 3: Cumulative distribution of expected H I optical depth measurements for an SKA1-SUR all-sky absorption survey. The histogram shows the number of optical depths per square degree we expect to measure to a given $\tau$ or smaller. These are based on detections in $0.3 \mathrm{~km} / \mathrm{s}$ channels where $\sigma_{S}=1.2 \mathrm{mJy}$.

Figure 4 shows the expected sky density of $\mathrm{H}$ I absorption measurements based on $\sigma_{\tau}$ and weighted by the $\mathrm{H}$ I column density. The left panel shows an estimate of the expected number of WNM measurements with $T_{s}>4000 \mathrm{~K}$ overlaid on an $\mathrm{H}$ I total column density image and the right panel shows an estimate of the number of CNM measurements with $T_{s}<500 \mathrm{~K}$. The accuracy of spin temperature measurements from $\mathrm{H}$ I absorption is limited by the error envelope imposed 

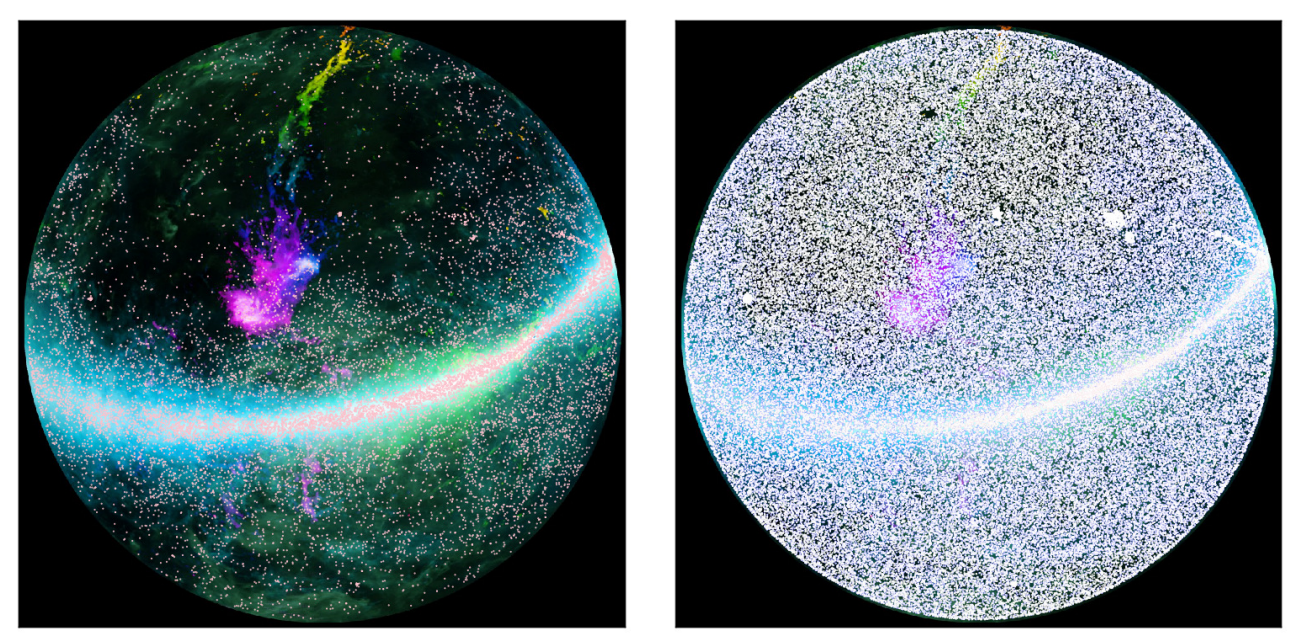

Figure 4: Source density of H I absorption measurements expected with SKA1 of the WNM (left) and the CNM (right). Each dot is an anticipated absorption measurement. The color image in the background is $\mathrm{H}$ I emission, where color represents velocity from the Parkes Galactic All-Sky Survey (McClure-Griffiths et al. 2009).

by emission fluctuations around a continuum source. It is therefore essential to have sufficient surface brightness sensitivity on angular scales $<30$ " to measure the fluctuations in emission. Extrapolating spatial power spectra currently measured in the Galactic plane and MCs (Elmegreen et al. 2001; Dickey et al. 2001) suggests that we will require $\sigma_{T}<1.7 \mathrm{~K}$ at 30 " to fully take advantage of the sensitivity of the absorption measurements.

The average linewidth, even for cold $\mathrm{H} \mathrm{I}$, will be $2.5 \mathrm{~km} \mathrm{~s}^{-1}$, so some gains can be achieved by averaging channels. Averaging to $\sim 1 \mathrm{~km} \mathrm{~s}^{-1}$ channels will give $\sigma_{\tau}<0.05$ towards more than 10 sources deg ${ }^{-2}$. If $\tau=N_{H I} /\left(1.8 \times 10^{18} \mathrm{~cm}^{-2} T_{s} * \Delta v\right)$, we can detect $N_{H I}=5.6 \times 10^{18} \mathrm{~cm}^{-2}$ in a $2.5 \mathrm{~km} \mathrm{~s}^{-1}$ line, assuming $T_{s}=100 \mathrm{~K}$. This will be an outstanding progression beyond the well known Millennium survey (Heiles \& Troland 2003, 2005), which has been the gold-standard for our knowledge of the distribution of temperatures in the MW neutral ISM with its measurement of 202 cold neutral components toward 79 high Galactic latitude sources.

Furthermore, the all-sky SKA1 H I absorption survey will measure absorption in the WNM. While the 21-SPONGE survey (Murray et al. 2014) will undoubtedly directly measure several WNM temperatures, the numbers of measurements before the SKA1 are likely to be of the order of tens. The SKA1-SUR all-sky absorption survey will be able to directly detect this WNM component towards hundreds of sources, as shown in Figures 3 and 4. Measurements of $\mathrm{H}$ I absorption will extend to HVCs, where there are currently only two (Wakker et al. 1991; Matthews et al. 2009). Given the sky density of background sources and the column density distribution of known HVCs (Moss et al. 2013), a blind all-sky survey covering all HVCs should detect cold H I components in absorption towards most known HVCs $\left(\delta<+30^{\circ}\right)$ with narrow line components and measure their spin temperatures.

For maximal efficiency of SKA1-SUR time this project should be conducted commensally with an all-sky continuum or extra-galactic $\mathrm{H}$ I survey, provided the correlator can be configured to provide $1.5 \mathrm{kHz}$ spectral channels over $\sim 5 \mathrm{MHz}$ around $1420 \mathrm{MHz}$. This could be achieved 
through either a "zoom-mode" or through narrow spectral channels over the entire range PAF frequency range. In this case, the Galactic $\mathrm{H}$ I absorption survey comes for free with the extragalactic H I survey.

\subsection{Targeted Galactic and Magellanic surveys}

To achieve the scientific goals of understanding the transition from atomic to molecular gas described in $\$ 2$ SKA1 should be used for a targeted survey of: (i) the MCs and (ii) the Galactic plane. The required sensitivity for these two regions is different, with the MCs requiring deeper integrations over a comparatively small area. Integrations of 200 hours on the MCs and 50-hours on the Galactic Plane will exceed current surveys and those and planned for the Australian SKA Pathfinder (ASKAP) by about an order of magnitude in sensitivity. The flux limits for these surveys are given in Table 3.

These surveys will deliver detailed H I emission images capable of measuring turbulence through the spatial power spectrum and probing the structure of $\mathrm{H}$ I flows around individual molecular clouds to AU scales in the MW and pc scales in the MCs. The data will be matched to the resolution of data available from space missions, such as Herschel, and molecular line measurements with ALMA. Imaging of all spatial scales, from $20^{\prime \prime}$ to many degrees, will be needed. To achieve this we will require short baselines as well as the ability to combine single dish data, as discussed below. With a spectral resolution of less than $1 \mathrm{~km} \mathrm{~s}^{-1}$ our spatially resolved $\mathrm{H}$ I images will trace velocity gradients in gas flow. As shown in Table 3 the survey will deliver $14 \mathrm{H} \mathrm{I}$ absorption sources per square degree across the Galactic plane and up to 25 per square degree in the MCs. These measurements will allow comprehensive measurements of the temperature distribution of gas across the Galaxy and MCs as well as detailed calibration of the H I self-absorption observed in $\mathrm{H}$ I emission images. To successfully remove the effects of $\mathrm{H}$ I emission fluctuations from the absorption spectra we will require $\sigma_{T} \sim 0.5 \mathrm{~K}$ at 30 ".

To achieve the needed sensitivity on the MCs $\left(100 \mathrm{deg}^{2}\right)$ with 200 hours dwell time per point gives a total of $2 \times 10^{4} \mathrm{deg}^{2} \mathrm{hr}$. The Galactic plane area $\left(|b|<2^{\circ}\right)$, is $1100 \mathrm{deg}^{2}$ so for 50 hours dwell time per point the total is $5.5 \times 10^{4} \mathrm{deg}^{2} \mathrm{hr}$. Dividing by the PAF FoV of SKA1-SUR at 1.4 $\mathrm{GHz}, \mathrm{FOV}=18 \mathrm{deg}^{2}$ gives a total survey time of about 125 days.

\subsection{Zero-spacing information}

Both the Galactic plane and all-sky surveys require the inclusion of so-called "zero-spacing", to recover the total flux and structure of the diffuse $\mathrm{H}$ I emission that fills the sky. The technical aspects of how this might be achieved with the SKA have to be considered carefully. Typically "zero-spacing" data are provided from single-dish surveys, which effectively sample baselines from $0 \mathrm{~m}$ to the size of the single dish. Provided these data are matched in spectral resolution and brightness sensitivity to the interferometric data and the interferometer has sufficient baselines smaller than the size of the single dish to allow for cross-calibration, the two datasets can be combined to provide an image sensitive to all angular scales larger than the resolution limit of the interferometer. For SKA Phase 1 the surface brightness sensitivity expected by even the deep Galactic plane ( $0.3 \mathrm{~K}$ per $0.3 \mathrm{~km} \mathrm{~s}^{-1}$ channel) is at the level that can attained by large all-sky single dish surveys similar to GASS (McClure-Griffiths et al. 2009; Kalberla et al. 2010) and EBHIS (Winkel et al. 
2010), which both reach $\sim 50 \mathrm{mK}$ sensitivities at $1 \mathrm{~km} \mathrm{~s}^{-1}$ spectral resolution. As SKA2 becomes available it may be necessary to use antennas of the SKA itself, through its autocorrelations or other means, to obtain short-spacings information at sufficient sensitivity. Although for SKA1 data can be obtained with existing single dish telescopes, it will be necessary to accommodate the data processing requirements of adding short-spacings in all stages of the SKA.

\subsection{Phasing in of SKA1}

Galactic fields will benefit from the sensitivity of a fully scoped SKA1 1 . The Galactic demands pull the SKA design to its two extremes: short baselines for surface brightness sensitivity and long baselines for $\mathrm{H} \mathrm{I}$ absorption. Given $\sim 50 \%$ less collecting area, it would be preferable to start with the shorter baselines as the survey speed for surface brightness sensitivity scales as the inverse of the square of the filling factor, or one over the longest baseline length to the fourth power. By contrast the survey speed for point source sensitivity has no dependence on baseline length and goes simply as the square of the collecting area. The scientific objectives are such that even for angular resolutions of 20 " at $50 \%$ of its baseline collecting area SKA1 would be an advance over ASKAP. For a phased SKA1 working for some time without the longest baselines $(<4000 \mathrm{~m})$ is satisfactory and could still provide an outstanding Galactic survey. Furthermore, emission line surveys of the targeted Galactic plane and MCs could be completed with worse angular resolution than the natural SKA1-SUR array, and still achieve more than a factor of five improvement in sensitivity at a factor of a few improvement in angular resolution over ASKAP.

\section{All-sky H I Emission and Absorption with the SKA}

The ultimate goal for SKA is a complete survey of Galactic H I in emission and absorption over $3 \pi$ steradians. All-sky Galactic $\mathrm{H}$ I emission surveys have been limited to single-dish surveys with angular resolutions of typically 10-60' (Winkel et al. 2010; Kalberla et al. 2010; McClure-Griffiths et al. 2009; Kalberla et al. 2005) and at best 3'(GALFA; Peek et al. 2011). From these all-sky surveys comes the bulk of our knowledge about the disk-halo interaction of the Milky Way, the overall structure of the Galaxy and the distribution of the phases. These provide essential foregrounds for interpreting observations of everything from X-ray sources to cosmological backgrounds. They have been essential to recent estimates of the total gas quantities in warm and cold $\mathrm{H}$ I and dust from comparisons with Planck (e.g. Planck collaboration 2013, Fukui et al. 2014). After very little improvement in angular resolution over the past 30 years, SKA offers us the first chance to conduct an all-sky MW survey at interferometric resolution, improving the angular resolution by more than an order of magnitude. A fully equipped SKA with 10 times the sensitivity of SKA1 will be almost unfathomably powerful, helping us to reach sub-Kelvin sensitivity over the whole local H I sky. Finally we will have the sensitivity to map the low-column density component of the high velocity sky in emission, while simultaneously measuring the fluctuations in $\mathrm{H} \mathrm{I}$ emission necessary to interpret $\mathrm{H}$ I absorption spectra. With these observations we will be able to realize all of the goals outlined in $\S 2$.

Together with the all-sky H I emission survey SKA will obtain measurements of optical depths, $\tau<10^{-2}$ towards half a million sources $\left(\sim 20 \mathrm{deg}^{-2}\right.$; Figure 2$)$, giving direct measurements of the 
temperature and column density of the CNM throughout the Galactic disk and halo. Most excitingly, the SKA all-sky HI absorption survey will be ably to detect the warm WNM in absorption towards all $>700$ mJy sources, giving $\sim 4000$ direct measurements of the temperature of the WNM. With these we will be able to determine the fractions of thermally stable and unstable gas in differing galactic environments. Finally we will be able to move beyond mapping to actually measuring the physical properties of H I throughout the MW and Magellanic System on the angular scale that we currently observe $\mathrm{H}$ I emission, $15^{\prime}$.

\section{Summary}

The SKA will revolutionize our knowledge of the evolution of the MW and the Magellanic System. As we move first through SKA1 towards SKA2 we will be able to image the structure and measure the physical properties of H I throughout the Milky Way, its halo and the Magellanic System. With these data we expect to reveal the multi-scale physics that determines structure formation in the MW, probe the transformation of atomic gas to molecular clouds and determine the gas accretion processes crucial to the long-term survival of the MW.

The nature of structure formation is a question that drives many fields of astrophysics, from the formation of galaxy clusters to the formation of planets. The processes driving structure formation are multi-scale by nature with sources and sinks of energy and mass at all scales. The multi-scale nature of the ISM spans more than eight orders of magnitude in spatial scale probing a tremendous range of physical processes (Armstrong et al. 1995). ISM studies with the multi-scale sensitivity of the SKA will provide hereto inaccessible insight into the detailed dynamical processes that govern the flux of mass and energy to and from various ISM phases. These are crucial to understanding the hydrogen cycle of galaxies, and in turn the evolution of structure in the Universe.

With the SKA we will have measurements of the thermal state, accretion rates and origins of gas traveling into and out of the MW. When combined with measurements of ionized gas we should finally be able to produce a full census of material in the halo of the MW, and relate the rate and efficiency of accretion to the rate of star formation within disk.

By studying $\mathrm{H}$ I in three different galactic laboratories (MW, Small and Large Magellanic Clouds) we will reveal how $\mathrm{H}$ I transforms into molecular clouds in regions with different metallicities and UV radiation fields. When compared with theoretical models of molecular cloud formation this will have important implications on the evolution of gas in a variety of cosmic environments.

\section{References}

Armstrong, J. W., Rickett, B. J., \& Spangler, S. R. 1995, ApJ, 443, 209

Audit, E., \& Hennebelle, P. 2005, A\&A, 433, 1

Ballesteros-Paredes, J., Hartmann, L., \& Vázquez-Semadeni, E. 1999, ApJ, 527, 285

Ballesteros-Paredes, J., Hartmann, L. W., Vázquez-Semadeni, E., Heitsch, F., \& Zamora-Avilés, M. A. 2011, MNRAS, 411, 65

Banerjee, R., Vázquez-Semadeni, E., Hennebelle, P., \& Klessen, R. S. 2009, MNRAS, 398, 1082

Ben Bekhti, N., Richter, P., Winkel, B., Kenn, F., \& Westmeier, T. 2009, A\&A, 503, 483

Bensby, T., Feltzing, S., \& Oey, M. S. 2014, A\&A, 562, A71 
Bhat, N. D. R., \& Gupta, Y. 2002, ApJ, 567, 342

Bigiel, F., Leroy, A., Walter, F., et al. 2008, AJ, 136, 2846

Braun, R. 1997, ApJ, 484, 637

Braun, R., 2014, “SKA1 Imaging Science Performance”, Document number SKA-TEL- SKO-DDXXX Revision A Draft 2

Braun, R., \& Kanekar, N. 2005, A\&A, 436, L53

Bregman, J. N. 1980, ApJ, 236, 577

Burkhart, B., Stanimirović, S., Lazarian, A., \& Kowal, G. 2010, ApJ, 708, 1204

Burstein, D., \& Heiles, C. 1978, ApJ, 225, 40

Carilli, C. L., Dwarakanath, K. S., \& Goss, W. M. 1998, ApJL, 502, L79

Chepurnov, A., Lazarian, A., Stanimirović, S., Heiles, C., \& Peek, J. E. G. 2010, ApJ, 714, 1398

Chiappini, C., Matteucci, F., \& Romano, D. 2001, ApJ, 554, 1044

Clark, P. C., Glover, S. C. O., Klessen, R. S., \& Bonnell, I. A. 2012, MNRAS, 424, 2599

Condon, J. J., Cotton, W. D., Greisen, E. W., et al. 1998, AJ, 115, 1693

Crovisier, J., \& Dickey, J. M. 1983, A\&A, 122, 282

de Avillez, M. A. 2000, MNRAS, 315, 479

Deshpande, A. A., Dwarakanath, K. S., \& Goss, W. M. 2000, ApJ, 543, 227

Dickey, J. M., \& Brinks, E. 1993, ApJ, 405, 153

Dickey, J. M., McClure-Griffiths, N. M., Stanimirović, S., Gaensler, B. M., \& Green, A. J. 2001, ApJ, 561, 264

Dickey, J. M., Mebold, U., Marx, M., et al. 1994, A\&A, 289, 357

Dickey, J. M., Mebold, U., Stanimirovic, S., \& Staveley-Smith, L. 2000, ApJ, 536, 756

Dickey, J. M., Salpeter, E. E., \& Terzian, Y. 1977, ApJL, 211, L77

Dickey, J. M., Strasser, S., Gaensler, B. M., et al. 2009, ApJ, 693, 1250

Dickman, R. L., \& Kleiner, S. C. 1985, ApJ, 295, 479

Dobbs, C. L., Pringle, J. E., \& Burkert, A. 2012, MNRAS, 425, 2157

Dwarakanath, K. S., Carilli, C. L., \& Goss, W. M. 2002, ApJ, 567, 940

Elmegreen, B. G., Kim, S., \& Staveley-Smith, L. 2001, ApJ, 548, 749

Elmegreen, B. G., \& Scalo, J. 2004, ARA\&A, 42, 211

Falceta-Gonçalves, D., Kowal, G., Falgarone, E., \& Chian, A. C.-L. 2014, Nonlinear Processes in Geophysics, 21, 587

Falgarone, E., Pety, J., \& Hily-Blant, P. 2009, A\&A, 507, 355

Fiedler, R. L., Dennison, B., Johnston, K. J., \& Hewish, A. 1987, Nature, 326, 675

Field, G. B. 1965, ApJ, 142, 531

Field, G. B., Goldsmith, D. W., \& Habing, H. J. 1969, ApJL, 155, L149

Fox, A. J., Wakker, B. P., Barger, K. A., et al. 2014, ApJ, 787, 147

Fraternali, F., Marasco, A., Marinacci, F., \& Binney, J. 2013, ApJ, 764, L21

Fukui, Y., Okamoto, R., Yamamoto, H., et al. 2014, ApJ, 796, 59

Gaensler, B. M., Tanna, A., Slane, P. O., et al. 2008, ApJ, 680, L37

Gaensler, B. M., Haverkorn, M., Burkhart, B., et al. 2011, Nature, 478, 214

Gazol, A., Vázquez-Semadeni, E., \& Kim, J. 2005, ApJ, 630, 911

Gazol, A., Vázquez-Semadeni, E., Sánchez-Salcedo, F. J., \& Scalo, J. 2001, ApJL, 557, L121

Gibson, S. J., Taylor, A. R., Higgs, L. A., Brunt, C. M., \& Dewdney, P. E. 2005, ApJ, 626, 195 
Glover, S. C. O., \& Mac Low, M.-M. 2007, ApJ, 659, 1317

Gomez, G. C., \& Vazquez-Semadeni, E. 2014, ApJ, 791, 124

Green, D. A. 1993, MNRAS, 262, 327

Green, G. M., Schlafly, E. F., Finkbeiner, D. P., et al. 2014, ApJ, 783, 114

Grenier, I. A., Casandjian, J.-M., \& Terrier, R. 2005, Science, 307, 1292

Heiles, C. 1997, ApJ, 481, 193

Heiles, C., \& Troland, T. H. 2003, ApJ, 586, 1067

—. 2005, ApJ, 624, 773

Heiner, J. S., Vázquez-Semadeni, E., \& Ballesteros-Paredes, J. 2014, ArXiv e-prints

Heitsch, F., Burkert, A., Hartmann, L. W., Slyz, A. D., \& Devriendt, J. E. G. 2005, ApJL, 633, L113

Heitsch, F., \& Hartmann, L. 2008, ApJ, 689, 290

Heitsch, F., Hartmann, L. W., Slyz, A. D., Devriendt, J. E. G., \& Burkert, A. 2008, ApJ, 674, 316

Heitsch, F., \& Putman, M. E. 2009, ApJ, 698, 1485

Heitsch, F., Slyz, A. D., Devriendt, J. E. G., Hartmann, L. W., \& Burkert, A. 2006, ApJ, 648, 1052 Hill, A. S., Joung, M. R., Mac Low, M.-M., et al. 2012, ApJ, 750, 104

Hopkins, A. M., McClure-Griffiths, N. M., \& Gaensler, B. M. 2008, ApJ, 682, L13

Huterer, D., Cunha, C. E., \& Fang, W. 2013, MNRAS, 432, 2945

Joung, M. R., Putman, M. E., Bryan, G. L., Fernández, X., \& Peek, J. E. G. 2012, ApJ, 759, 137

Kalberla, P. M. W., Burton, W. B., Hartmann, D., et al. 2005, A\&A, 440, 775

Kalberla, P. M. W., \& Dedes, L. 2008, A\&A, 487, 951

Kalberla, P. M. W., \& Haud, U. 2006, A\&A, 455, 481

Kalberla, P. M. W., Schwarz, U. J., \& Goss, W. M. 1985, A\&A, 144, 27

Kalberla, P. M. W., McClure-Griffiths, N. M., Pisano, D. J., et al. 2010, A\&A, 521, 17

Kennicutt, Jr., R. C. 1998, ApJ, 498, 541

Kim, C.-G., Ostriker, E. C., \& Kim, W.-T. 2014, ApJ, 786, 64

Kim, S., Staveley-Smith, L., Dopita, M. A., et al. 1998, ApJ, 503, 674

Kritsuk, A. G., Norman, M. L., Padoan, P., \& Wagner, R. 2007, ApJ, 665, 416

Krumholz, M. R., McKee, C. F., \& Tumlinson, J. 2009, ApJ, 693, 216

Krčo, M., Goldsmith, P. F., Brown, R. L., \& Li, D. 2008, ApJ, 689, 276

Langer, W. D., Velusamy, T., Pineda, J. L., et al. 2010, A\&A, 521, L17

Larson, R. B. 1981, MNRAS, 194, 809

Lazarian, A., \& Pogosyan, D. 2000, ApJ, 537, 720

Lee, M.-Y., Stanimirović, S., Douglas, K. A., et al. 2012, ApJ, 748, 75

Lehner, N., Howk, J. C., Thom, C., et al. 2012, MNRAS, 424, 2896

Leroy, A., Bolatto, A., Stanimirovic, S., et al. 2007, ApJ, 658, 1027

Levine, E. S., Blitz, L., \& Heiles, C. 2006, ApJ, 643, 881

Li, D., \& Goldsmith, P. F. 2003, ApJ, 585, 823

Li, D., \& Goldsmith, P. F. 2012, ApJ, 756, 12

Liszt, H. 2001, A\&A, 371, 698

Lockman, F. J. 1984, ApJ, 283, 90

Mac Low, M.-M., McCray, R., \& Norman, M. L. 1989, ApJ, 337, 141

Matthews, D., Staveley-Smith, L., Dyson, P., \& Muller, E. 2009, ApJL, 691, L115 
McClure-Griffiths, N. M., Ford, A., Pisano, D. J., et al. 2006, ApJ, 638, 196

McClure-Griffiths, N. M., Pisano, D. J., Calabretta, M. R., et al. 2009, ApJS, 181, 398

McKee, C. F., \& Ostriker, E. C. 2007, ARA\&A, 45, 565

McKee, C. F., \& Ostriker, J. P. 1977, ApJ, 218, 148

Meixner, M., Panuzzo, P., Roman-Duval, J., et al. 2013, AJ, 146, 62

Moss, V. A. 2014, PhD Thesis

Moss, V. A., McClure-Griffiths, N. M., Murphy, T., et al. 2013, ApJS, 209, 12

Muller, E., Staveley-Smith, L., Zealey, W., \& Stanimirović, S. 2003, MNRAS, 339, 105

Murray, C. E., Lindner, R. R., Stanimirović, S., et al. 2014, ApJ, 781, L41

Mutch, S. J., Croton, D. J., \& Poole, G. B. 2011, ApJ, 736, 84

Nigra, L., Stanimirović, S., Gallagher, III, J. S., et al. 2012, ApJ, 760, 48

Ntormousi, E., Burkert, A., Fierlinger, K., \& Heitsch, F. 2011, ApJ, 731, 13

Peek, J. E. G. 2013, ApJL, 766, L6

Peek, J. E. G., Heiles, C., Douglas, K. A., et al. 2011, ApJS, 194, 20

Pidopryhora, Y., Lockman, F. J., \& Shields, J. C. 2007, ApJ, 656, 928

Pingel, N. M., Stanimirović, S., Peek, J. E. G., et al. 2013, ApJ, 779, 36

Planck Collaboration, Ade, P. A. R., Aghanim, N., et al. 2011, Astronomy and Astrophysics, 536, A19

Planck Collaboration, Abergel, A., Ade, P. A. R., et al. 2013, ArXiv e-prints

Putman, M. E., de Heij, V., Staveley-Smith, L., et al. 2002, AJ, 123, 873

Putman, M. E., Peek, J. E. G., \& Joung, M. R. 2012, ARA\&A, 50, 491

Qian, L., Li, D., \& Goldsmith, P. F. 2012, ApJ, 760, 147

Reynolds, R. J. 1991, in IAU Symp. 144: The Interstellar Disk-Halo Connection in Galaxies, ed. H. Bloeman, 67-76

Richter, P., Sembach, K. R., Wakker, B. P., \& Savage, B. D. 2001, ApJL, 562, L181

Rickett, B. J. 1977, ARA\&A, 15, 479

Roy, N., Minter, A. H., Goss, W. M., Brogan, C. L., \& Lazio, T. J. W. 2012, ApJ, 749, 144

Schlafly, E. F., Green, G., Finkbeiner, D. P., et al. 2014, ApJ, 789, 15

Schlegel, D. J., Finkbeiner, D. P., \& Davis, M. 1998, ApJ, 500, 525

Schönrich, R., \& Binney, J. 2009, MNRAS, 399, 1145

Schruba, A., Leroy, A. K., Walter, F., et al. 2011, AJ, 142, 37

Shapiro, P. R., \& Field, G. B. 1976, ApJ, 205, 762

Smith, R. J., Glover, S. C. O., Clark, P. C., Klessen, R. S., \& Springel, V. 2014, MNRAS, 441, 1628

Stanimirović, S., Hoffman, S., Heiles, C., et al. 2008, ApJ, 680, 276

Stanimirović, S., \& Lazarian, A. 2001, ApJL, 551, L53

Stanimirović, S., Staveley-Smith, L., Dickey, J. M., Sault, R. J., \& Snowden, S. L. 1999, MNRAS, 302,417

Strasser, S. T., Dickey, J. M., Taylor, A. R., et al. 2007, AJ, 134, 2252

Thom, C., Peek, J. E. G., Putman, M. E., et al. 2008, ApJ, 684, 364

van Dishoeck, E. F., \& Black, J. H. 1988, ApJ, 334, 771

Vázquez-Semadeni, E., Gazol, A., \& Scalo, J. 2000, ApJ, 540, 271

Vázquez-Semadeni, E., Gómez, G. C., Jappsen, A. K., et al. 2007, ApJ, 657, 870 
Vázquez-Semadeni, E., Ryu, D., Passot, T., González, R. F., \& Gazol, A. 2006, ApJ, 643, 245

Veilleux, S., Cecil, G., \& Bland-Hawthorn, J. 2005, ARA\&A, 43, 769

Vogelsberger, M., Genel, S., Springel, V., et al. 2014, Nature, 509, 177

Wakker, B. P., \& Mathis, J. S. 2000, ApJL, 544, L107

Wakker, B. P., Vijfschaft, B., \& Schwarz, U. J. 1991, A\&A, 249, 233

Walker, M., \& Wardle, M. 1998, ApJL, 498, L125

Werk, J. K., Prochaska, J. X., Tumlinson, J., et al. 2014, ArXiv e-prints

Westerlund, B. E. 1997, The Magellanic Clouds (Cambridge University Press)

Winkel, B., Kalberla, P. M. W., Kerp, J., \& Flöer, L. 2010, ApJS, 188, 488

Wolfire, M. G., Hollenbach, D., McKee, C. F., Tielens, A. G. G. M., \& Bakes, E. L. O. 1995, ApJ, 443,152

Wolfire, M. G., McKee, C. F., Hollenbach, D., \& Tielens, A. G. G. M. 2003, ApJ, 587, 278 